\title{
Gravitational waves from very massive stars collapsing to a black hole
}

\author{
Haruki Uchida, ${ }^{1}$ Masaru Shibata, ${ }^{2,1}$ Koh Takahashi, ${ }^{3}$ and Takashi Yoshida ${ }^{4}$ \\ ${ }^{1}$ Yukawa Institute for Theoretical Physics, Kyoto University, Kyoto, 606-8502, Japan \\ ${ }^{2}$ Max Planck Institute for Gravitational Physics (Albert Einstein Institute), \\ Am Muhlenberg 1, Potsdam-Golm 14476, Germany \\ ${ }^{3}$ Argelander-Institut für Astronomie, Universiät Bonn, D-53121 Bonn, Germany \\ ${ }^{4}$ Department of Astronomy, Graduate School of Science, \\ the University of Tokyo, Tokyo, 113-0033, Japan
}

(Dated: January 25, 2019)

\begin{abstract}
We compute gravitational waves emitted by the collapse of a rotating very massive star (VMS) core leading directly to a black hole in axisymmetric numerical-relativity simulations. The evolved rotating VMS is derived by a stellar evolution calculation and its initial mass and the final carbonoxygen core mass are $320 M_{\odot}$ and $\approx 150 M_{\odot}$, respectively. We find that for the moderately rapidly rotating cases, the peak strain amplitude and the corresponding frequency of gravitational waves are $\sim 10^{-22}$ and $f \approx 300-600 \mathrm{~Hz}$ for an event at the distance of $D=50 \mathrm{Mpc}$. Such gravitational waves will be detectable only for $D \lesssim 10 \mathrm{Mpc}$ by second generation detectors, advanced LIGO, advanced VIRGO, and KAGRA, even if the designed sensitivity for these detectors is achieved. However, thirdgeneration detectors will be able to detect such gravitational waves for an event up to $D \sim 100 \mathrm{Mpc}$. The detection of the gravitational-wave signal will provide a potential opportunity for verifying the presence of VMSs with mass $\gtrsim 300 M_{\odot}$ and their pair-unstable collapse in the universe.
\end{abstract}

PACS numbers: 04.25.D-, 04.30.-w, 04.40.Dg

\section{INTRODUCTION}

Gravitational collapse of a carbon-oxygen (CO) core induced by the instability associated with the electronpositron pair creation (i.e., pair instability) is a possible fate of a very massive star (VMS) of initial mass $\gtrsim 140 M_{\odot}$ [1] 4$]$. Broadly speaking, there are two possible fates for the pair-instability collapse of the VMSs. For the relatively low-mass case, oxygen burning occurs explosively during the collapse, and then, the thermal pressure resulting from the thermal energy released by the thermal nuclear reaction of oxygen halts the collapse, leading to a pair-instability supernova explosion (PISN). On the other hand, for the high-mass case, the collapse cannot be halted by the nuclear burning, and hence, the final remnant is a black hole $(\mathrm{BH})$ possibly surrounded by a disk and an outflow [5]. However, PISN and/or BH formation have not been confirmed yet.

In this article, we report a result of our new numericalrelativity simulations for the collapse of a rotating VMS of its initial mass $320 M_{\odot}$ to a $\mathrm{BH}$, paying attention to gravitational waves emitted during the $\mathrm{BH}$ formation. We show that (i) the gravitational-wave signal is characterized by a ringdown oscillation of the newlyformed $\mathrm{BH}$; (ii) for the moderately rapidly rotating cases, the energy emitted by gravitational waves is typically $2 \times 10^{-7} M_{\mathrm{CO}} c^{2}$ where $M_{\mathrm{CO}}$ is the mass of the $\mathrm{CO}$ core just before the onset of the collapse; (iii) the frequency for the peak amplitude of gravitational waves is $300-600 \mathrm{~Hz}$ for $M_{\mathrm{CO}} \approx 150 M_{\odot}$ with the peak amplitude $\sim 10^{-22}$ for a hypothetical distance to sources $D \sim 50 \mathrm{Mpc}$. Such gravitational waves can be a target of third-generation gravitational-wave detectors such as Einstein Telescope [7].
This paper is organized as follows. In Sec. II, we summarize the setup of our numerical-relativity simulation. In Sec. III, we present the gravitational waveforms to show that these gravitational waves will be a source for third-generation gravitational-wave detectors. Section IV is devoted to a summary and discussion. Throughout this paper, $c$ and $G$ denote the speed of light and gravitational constant, respectively.

\section{SETUP}

Following Ref. [5], we employ an evolved rotating VMS derived by a stellar evolution code of Refs. [4, 8] for preparing the initial conditions for a numerical-relativity simulation. The stellar evolution calculation is performed for a metal-free star of its mass $320 M_{\odot}$ and of the rigid rotation with the angular velocity of $2.1 \times 10^{-4} \mathrm{~s}^{-1}$ at the zero-age main sequence stage. The calculation is continued until the central temperature reaches $\approx 10^{9.2} \mathrm{~K}$ for which the star is composed of a CO core (mainly of oxygen) and an envelope of helium and hydrogen (see Fig. 2 of Ref. [5]). At this stage, the total mass of the entire star reduces to $\approx 290 M_{\odot}$ because of the mass loss during the stellar evolution [6], and the mass of the CO core is $\approx 150 M_{\odot}$. The dimensionless spin parameter, $c J_{\mathrm{CO}} /\left(G M_{\mathrm{CO}}^{2}\right)$, of the $\mathrm{CO}$ core is $\approx 1.1$.

In this paper, we explore the collapse of the $\mathrm{CO}$ core, reducing its angular velocity uniformly to focus on the collapse of stellar cores for which the dimensionless spin

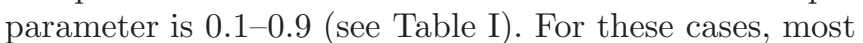
of the matter in the $\mathrm{CO}$ core eventually collapses into a rotating $\mathrm{BH}$. In the following, we pay particular attention to gravitational waves at the formation of the $\mathrm{BH}$, which 
TABLE I. Quantities for the CO core of VMS employed in this paper. $M_{\mathrm{CO}}$ : the mass. $\beta$ : ratio of rotational kinetic energy to gravitational potential energy. $J_{\mathrm{CO}}$ : angular momentum and $\chi:=c J_{\mathrm{CO}} /\left(G M_{\mathrm{CO}}^{2}\right)$. $R_{\mathrm{CO}}$ : equatorial radius.

\begin{tabular}{ccccc}
\hline Model & $M_{\mathrm{CO}}\left(M_{\odot}\right)$ & $\beta$ & $\chi$ & $R_{\mathrm{CO}}(\mathrm{km})$ \\
\hline M01 & 150 & $2.7 \times 10^{-5}$ & 0.11 & $6 \times 10^{5}$ \\
M03 & 150 & $2.4 \times 10^{-4}$ & 0.33 & $6 \times 10^{5}$ \\
M05 & 150 & $6.6 \times 10^{-4}$ & 0.55 & $6 \times 10^{5}$ \\
M07 & 150 & $1.3 \times 10^{-3}$ & 0.77 & $6 \times 10^{5}$ \\
M08 & 150 & $1.7 \times 10^{-3}$ & 0.88 & $6 \times 10^{5}$ \\
\hline
\end{tabular}

reflect the early formation process of the $\mathrm{BH}$.

Our method for the solution of Einstein's equation is the same as in Ref. [5]. We employ the original version of Baumgarte-Shapiro-Shibata-Nakamura formulation with a puncture gauge [10]. The gravitational field equations are solved in the standard 4th-order finite differencing scheme. The axial symmetry is imposed using a 4thorder cartoon method [5, 11, 12]. Gravitational waves are extracted from the outgoing-component of the complex Weyl scalar $\Psi_{4}$, which is expanded by a spin-weighted spherical harmonics of weight $-2,{ }_{-2} Y_{l m}(\theta, \varphi)$, with $m=$ 0 in axisymmetric spacetime (see, e.g., Ref. [13]). We focus only on the quadrupole mode with $l=2$ (denoted by $\Psi_{20}$ in the following) because it is the dominant mode (we checked that the amplitude of $l=3$ and 4 modes is much smaller than $l=2$ mode).

Formation of $\mathrm{BH}$ is determined by the presence of an apparent horizon. The mass and dimensionless spin of the $\mathrm{BH}$ are determined by measuring the area and circumferential radii of the apparent horizon (e.g., see Ref. 14] for our method).

Following Ref. [5], we use one of Timmes \& Swesty equations of state which includes the contribution from radiation, ions as ideal gas, electrons, positrons and corrections for Coulomb effects. For electrons and positrons, the relativistic effect, the effects of degeneration and electron-positron pair creation are taken into account. We also take into account the nuclear burning, photodissociation of iron and helium, and neutrino cooling.

The effect of the neutrino cooling is incorporated in the equation of motion as

$$
\nabla_{\alpha} T^{\alpha \beta}=\rho u^{\beta} q_{\text {neu }} f(\rho),
$$

where $\nabla_{\alpha}$ is the covariant derivative with respect to the spacetime metric, $T^{\alpha \beta}$ the energy-momentum tensor, $\rho$ the rest-mass density, $u^{\alpha}$ the four-velocity of the fluid, $q_{\text {neu }}$ the neutrino emission rate of Ref. [15], and $f(\rho)$ a function of $\rho$, respectively. Neutrinos freely escape from the collapsing core for the case that the density and temperature are not very high. However, for $\rho \gtrsim 10^{11} \mathrm{~g} / \mathrm{cm}^{3}$ with the temperature $T \gtrsim 5 \mathrm{MeV}$, neutrinos with their cross section $\gtrsim 6 \times 10^{-43}(T / 5 \mathrm{MeV})^{2} \mathrm{~cm}^{2}$ should be trapped in the collapsing core of radius $\sim 10^{7} \mathrm{~cm}$. To approximately take into account this effect, we introduce a function, $f(\rho)$, and set it as $\exp \left(-\rho / \rho_{0}\right)$ where $\rho_{0}$ is a constant set to be $10^{11} \mathrm{~g} / \mathrm{cm}^{3}$ at the fiducial runs. In addition, we perform simulations for model M07 with $\rho_{0}=10^{10} \mathrm{~g} / \mathrm{cm}^{3}$ and $\rho_{0}=0$ (i.e., $f=0$ ) to show that the effect of the neutrino cooling plays a key role for determining the spectrum of gravitational waves.

Numerical simulations are performed in cylindrical coordinates $(X, Z)$, and a nonuniform grid is used for $X$ and $Z$. Specifically, we employ the following grid spacing (the same profile is chosen for $X$ and $Z$ ): for $X_{i} \leq X_{\text {in }}, \Delta X=\Delta X_{0}=$ const and for $X_{i}>X_{\text {in }}$, $\Delta X_{i}=\eta \Delta X_{i-1} . \Delta X_{0}$ is the grid spacing in an inner region and $\Delta X_{i}:=X_{i+1}-X_{i}$ with $X_{i}$ the location of $i$ th grid. $\eta$ determines the nonuniform degree of the grid spacing for which we always choose 1.014. As in Ref. [5], for the early stage of the collapse, we employ large values of $\Delta X_{0}$ and $X_{\text {in }}$ for which we assign the same values as before [5], and then we perform a regriding for a better resolved simulation. For this later phase, we employ a grid resolution better than in our previous study 5] as $\Delta X_{0}=0.0048-0.0096 G M_{\mathrm{CO}} / c^{2}(\approx 1.1-2.2) \mathrm{km}$. Вy varying $\Delta X_{0}$ for such a range, we confirmed that the convergence of the gravitational waveform is well achieved.

\section{NUMERICAL RESULTS}

\section{A. Black hole formation processes}

For all the models considered in this paper, a $\mathrm{BH}$ is formed in the $\mathrm{CO}$ core collapse. However, the formation and evolution processes of the $\mathrm{BH}$ depend strongly on the effect of the nuclear reaction and neutrino cooling.

The left panel of Fig. 1 shows the evolution of the mass and dimensionless spin for M07 models with no nuclear reaction and $f(\rho)=0$ (no neutrino cooling), and with the nuclear reaction and various values of $\rho_{0}, 0,10^{10}$, and $10^{11} \mathrm{~g} / \mathrm{cm}^{3}$. This shows that for no nuclear reaction, a substantial fraction of the $\mathrm{CO}$ core collapses into a $\mathrm{BH}$ in the first $\sim 50 \mathrm{~ms}$. By contrast, in the presence of the nuclear reaction (i.e., in the more realistic case), the initial mass of the $\mathrm{BH}$ is much smaller than the total mass of the $\mathrm{CO}$ core. The reason for this is that the photodissociation of iron and helium significantly reduces the thermal pressure in the central region of the collapsing core, and as a result, the collapse of the central region is significantly accelerated, leading to a runaway collapse.

In addition to the nuclear reaction, the neutrino cooling plays an important role in determining the initial $\mathrm{BH}$ mass, $M_{i}$. For no neutrino cooling (i.e., $f(\rho)=0$ or $\left.\rho_{0}=0\right), M_{i} \sim 40 M_{\odot}$. On the other hand, if we take into account the neutrino cooling $\left(\rho_{0}=10^{10}\right.$ and $\left.10^{11} \mathrm{~g} / \mathrm{cm}^{3}\right)$, $M_{i}$ is smaller as $\sim 20-30 M_{\odot}$; i.e., for the larger value of $\rho_{0}, M_{i}$ is smaller. The reason for this is that by the neutrino cooling, the collapse is further accelerated in the central region, leading to a smaller initial $\mathrm{BH}$ mass.

By contract to the $\mathrm{BH}$ mass, the initial dimensionless spin is in a fairly narrow range between 0.53 and 0.68 , and 

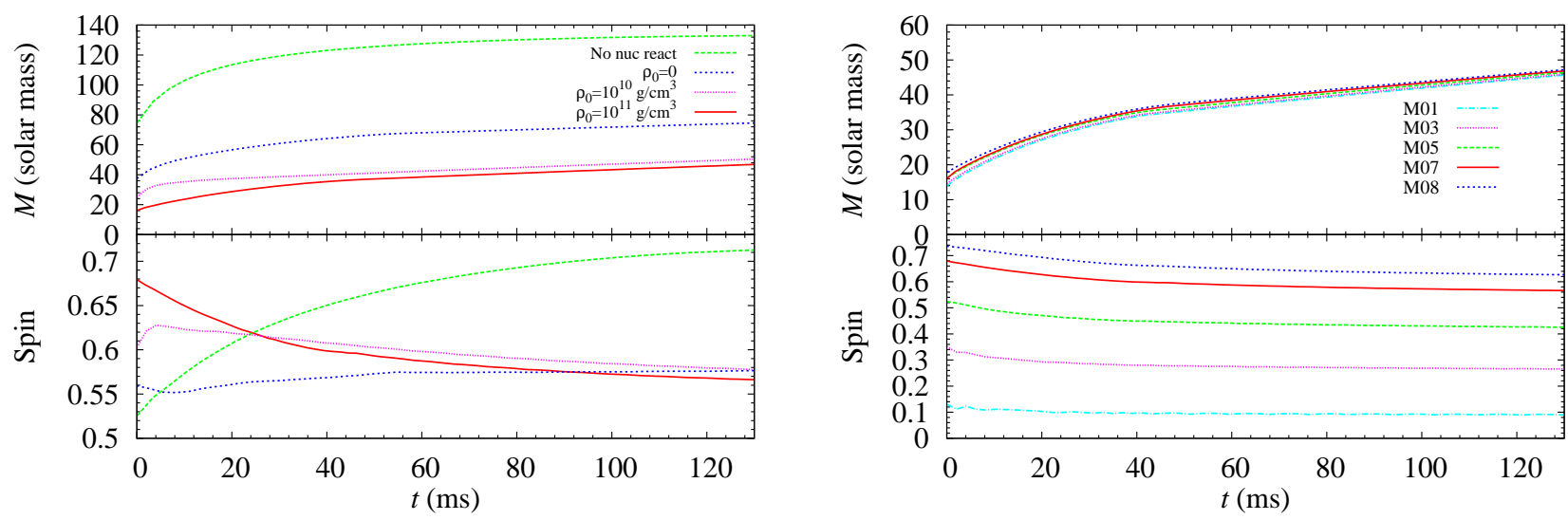

FIG. 1. Left: Evolution of mass and dimensionless spin of BH formed after the collapse of VMSs for M07 models. The dashed green, dotted blue, short-dotted magenta, and solid red curves show the results for no nuclear reaction and for the presence of nuclear reaction with $\rho_{0}=0,10^{10}$, and $10^{11} \mathrm{~g} / \mathrm{cm}^{3}$, respectively. We note that for the models in which the neutrino cooling is more efficient (for larger values of $\rho_{0}$ ), the initial BH mass is smaller. Right: The same as the left panel but for models M01, M03, M05, M07, and M08 in the presence of the nuclear reaction and the neutrino cooling with $\rho_{0}=10^{11} \mathrm{~g} / \mathrm{cm}^{3}$.

thus, it does not depend strongly on the effects of nuclear reaction and neutrino cooling for the M07 models. This property simply reflects the initial angular momentum distribution of the $\mathrm{CO}$ core at the onset of the collapse.

The right panel of Fig. 1 shows the evolution of the mass and dimensionless spin for models M01, M03, M05, M07, and M08 with the nuclear reaction and with the neutrino cooling for $\rho_{0}=10^{11} \mathrm{~g} / \mathrm{cm}^{3}$. This illustrates that the evolution of the $\mathrm{BH}$ mass depends only weakly on the total angular momentum of the collapsing star. On the other hand, the $\mathrm{BH}$ spin naturally reflects the angular momentum distribution of the $\mathrm{CO}$ cores.

\section{B. Gravitational waves}

Figure 2 displays gravitational waveforms $\left(\Psi_{20}\right)$ as a function of retarded time. Here, the time at which the maximum amplitude is reached is chosen as the origin of the time. We note that $l=2, m=0$ mode is proportional to $\sin ^{2} \theta$ where $\theta$ is the angle from the rotation axis. Thus, the amplitude becomes maximum for an observer located along the equatorial plane and it vanishes if the observer is located along the rotation axis. Figure 2 plots the waveforms for $\theta=\pi / 2$.

Figure 2 shows that gravitational waves are composed of a short precursor and ringdown oscillation associated with the formed $\mathrm{BH}$ for all the models considered in this paper. The period of the ringdown oscillation, $\lambda$, is varied for each model. We note that for the $l=2, m=0$ mode, $\lambda$ should be $\approx 16 G M_{\mathrm{BH}} / c^{3} \approx 1.6\left(M_{\mathrm{BH}} / 20 M_{\odot}\right) \mathrm{ms}$, where $M_{\mathrm{BH}}$ is the mass of the $\mathrm{BH}$, according to the linearperturbation analysis for the $\mathrm{BH}$ quasi-normal modes [16] with the dimensionless spin, $\chi_{\mathrm{BH}}=0-0.8$. For all the models with the nuclear reaction, $\lambda$ agrees approximately with that predicted by the $\mathrm{BH}$ perturbation theory if we take the initial mass of the $\mathrm{BH}$ as $M_{\mathrm{BH}}$. Thus, the gravitational waveforms reflect the early formation process of the BH. For the model with no nuclear reaction (dashed green curve in the left panel of Fig. 2), $\lambda \sim 16 \times\left(100 G M_{\odot} / c^{3}\right) \sim 8 \mathrm{~ms}$, reflecting the fact that a substantial fraction of the $\mathrm{CO}$ core matter simultaneously collapses into the BH.

The right panel of Fig. 2 shows that the gravitational waveforms depend weakly on the $\mathrm{BH}$ spin for $\chi \gtrsim 0.5$. The main reason for this is that the evolution process of the BH mass depends only weakly on the spin. For $\chi \lesssim 0.3$, the maximum amplitude decreases steeply with the decrease of $\chi$. This reflects the fact that for such small values of $\chi$, the degree of axial symmetry decreases with $\chi$. For higher $\mathrm{BH}$ spins, the damping time for the ringdown oscillation is longer. This also agrees with the prediction by the $\mathrm{BH}$ perturbation theory [16]. For $\chi \gtrsim$ 0.5 , the peak amplitude is slightly higher for the lower spin. This reflects the fact that for the higher spin, the collapse is decelerated by a stronger centrifugal force, and the gravitational-wave emission is slightly suppressed.

The total energy of gravitational waves emitted is $\Delta E \approx 7 \times 10^{-7} M_{\mathrm{CO}} c^{2}$ for the model with no nuclear reaction and $\approx 2 \times 10^{-7} M_{\mathrm{CO}} c^{2}$ for the models with the nuclear reaction and $\chi \gtrsim 0.5$. Thus, the emissivity is suppressed in the presence of the nuclear reaction (specifically photo-dissociation). The emissivity is much smaller than those in binary BH mergers in which $\sim 10 \%$ of the total mass energy can be radiated (e.g., Ref. [17]). The emissivity for no nuclear reaction is as large as that for the collapse of supermassive stars to a $\mathrm{BH}[18]$. For $\chi \lesssim 0.5, \Delta E$ steeply decreases with $\chi$ : $\Delta E /\left(M_{\mathrm{CO}} c^{2}\right) \approx 4 \times 10^{-9}$ and $1 \times 10^{-7}$ for M01 and M03.

Figure 3 shows a spectrum (an effective amplitude) of gravitational waves for $D=50 \mathrm{Mpc}$ for M07 models. 

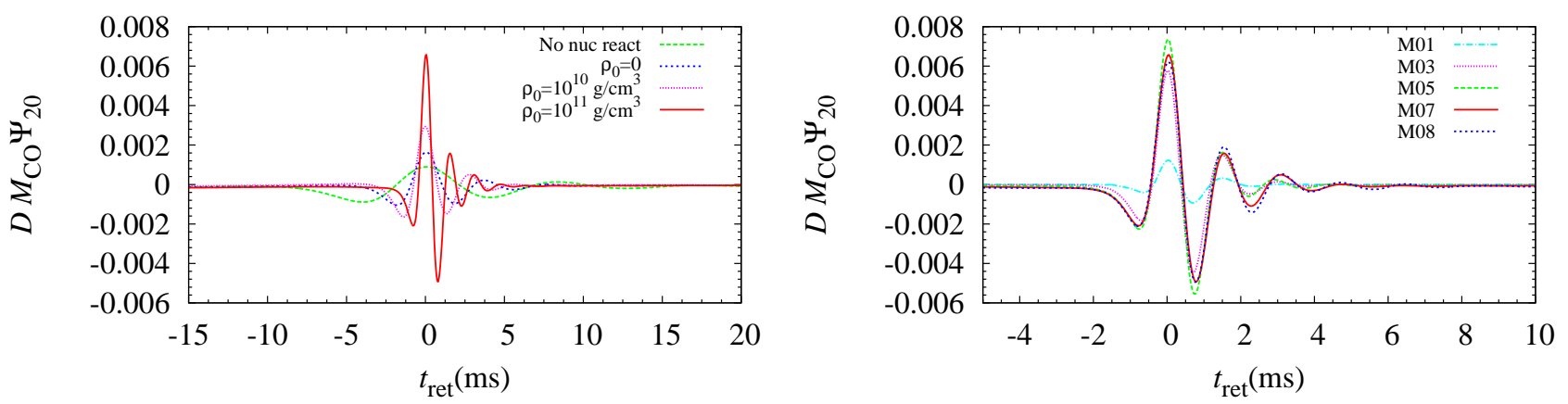

FIG. 2. Left: Gravitational waveforms $\left(l=2\right.$ axisymmetric mode of $\left.\Psi_{4}\right)$ as a function of retarded time for M07 models with no nuclear reaction (green dashed curve) and with nuclear reaction and neutrino cooling $\left(\rho_{0}=10^{11} \mathrm{~g} / \mathrm{cm}^{3}: \mathrm{solid} \mathrm{red} \mathrm{curve}\right.$, $\rho_{0}=10^{10} \mathrm{~g} / \mathrm{cm}^{3}$ : short-dotted magenta curve, and $\rho_{0}=0$ : dotted blue curve). Right: The same as the left panel but for models M01, M03, M05, M07, and M08 in the presence of the nuclear reaction and the neutrino cooling with $\rho_{0}=10^{11} \mathrm{~g} / \mathrm{cm}^{3}$. For these figures, the time at which the maximum amplitude is reached is chosen as the origin of the time to align the waveforms.
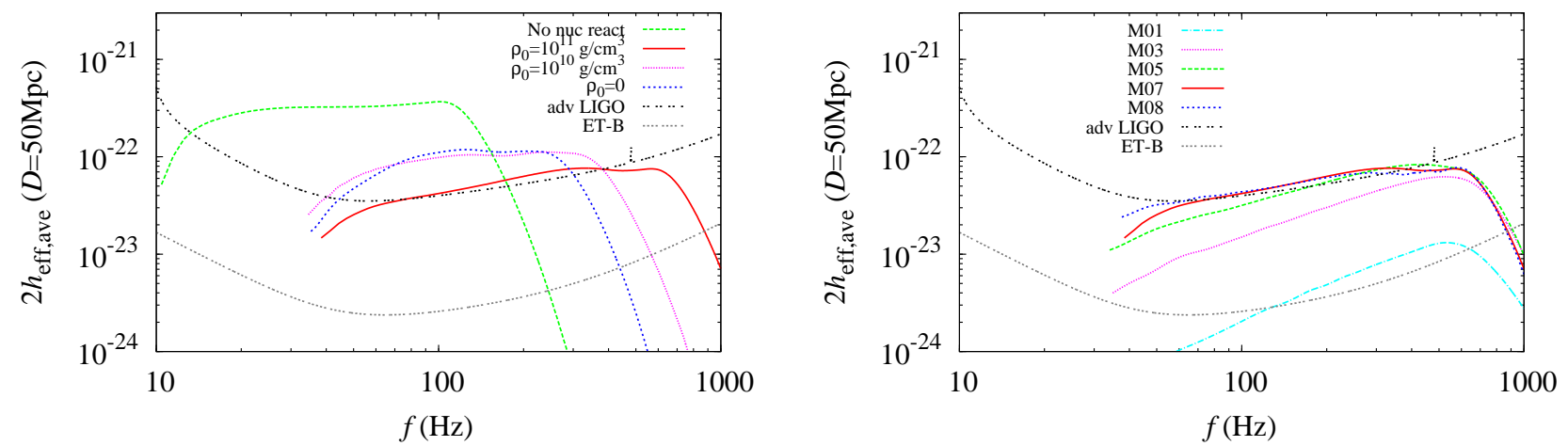

FIG. 3. Left: Fourier spectrum of gravitational waves for a hypothetical distance to the source $D=50 \mathrm{Mpc}$ for M07 models. Here, we plot $2 h_{\text {eff } \text {,ave }}=2 h_{\text {eff }} \times 2 / 3$. Each line type denotes the same model as in the left panel of Fig. 2 . The black dot-dot and grey dot-dot-dot curves show the design sensitivities of the advanced LIGO (the "Zero Detuning High Power" configuration [19]) and Einstein Telescope of the type B [20]. Right: The same as the left panel but for models M01, M03, M05, M07, and M08. Each line type denotes the same model as in the right panel of Fig. 2.

Here, the Fourier spectrum $h(f)$ is first derived from [13]

$$
h(f)=-_{-2} Y_{20} \int_{-t_{i}}^{t_{f}} d t \frac{2 \Psi_{20}(t)}{(2 \pi f)^{2}} \exp (-2 \pi i f t),
$$

where $t_{i}=t_{f} \approx 10-30 \mathrm{~ms}$ for the choice of time in which the maximum of $\Psi_{20}$ is reached at $t=0$. Here, $t_{i}$ and $t_{f}$ are varied depending on the characteristic wavelength of gravitational waves. Since ${ }_{-2} Y_{20}$ is proportional to $\sin ^{2} \theta$, the average of $h(f)$ with respect to $\theta$ is $h_{\text {ave }}(f)=$ $2 h(f)_{\theta=\pi / 2} / 3$. Then, we define the effective amplitude by $h_{\text {eff, ave }}:=f\left|h_{\text {ave }}(f)\right|$ [21]. In Fig. 3, we, in addition, multiply 2 because the signal-to-noise ratio (SNR) for a spectrum of gravitational waves, $g(f)$, is written as

$$
\mathrm{SNR}=\int_{0}^{\infty} d f \frac{(2|g(f)|)^{2}}{S_{n}(f)}
$$

where $S_{n}(f)$ is the one-sided noise spectrum density of a gravitational-wave detector (i.e., for each frequency $f, 2|f h(f)| / \sqrt{S_{n}(f) f}$ approximately denotes the SNR). The black dot-dot and grey dot-dot-dot curves show the design sensitivities of advanced LIGO (the "Zero Detuning High Power" configuration [19]) and Einstein Tele- scope of the type B 20]. Here, we plot a dimensionless quantity $\left(S_{n} f\right)^{1 / 2}$. We note that a low frequency part of $h(f)$ depends on the choice of $t_{i}$ and $t_{f}$, and hence, we do not plot the unreliable part of $h_{\mathrm{eff}}$,ave in Fig. 3 ,

Figure 3 shows that the peak amplitude of $h_{\mathrm{eff}}$,ave is proportional approximately to the initial mass of the formed $\mathrm{BH}$. On the other hand, the highest frequency for the peak amplitude is inversely proportional to the $\mathrm{BH}$ mass. Broadly speaking, for the initial $\mathrm{BH}$ mass, $M_{i}$, the maximum value of $2 h_{\mathrm{eff}}$,ave is written as $\sim 10^{-22}\left(M_{i} / 30 M_{\odot}\right)$ and the corresponding highest frequency is $\sim 400\left(M_{i} / 30 M_{\odot}\right)^{-1} \mathrm{~Hz}$. As indicated from the right panel of Fig. 2, these values depend very weakly on the angular momentum of the CO cores for $\chi \gtrsim 0.5$.

For the curves shown in Fig. 3, the SNR for the most optimistic alignment of a detector with respect to the direction of the source (see [21] for a remark) is calculated for $D=50 \mathrm{Mpc}$. For the sensitivity of advanced LIGO, it becomes $\approx 11$ for the model with no nuclear reaction and no neutrino cooling, $\approx 3.1$ for the models with the nuclear reaction and $\rho_{0}=0$ and $10^{10} \mathrm{~g} / \mathrm{cm}^{3}$, and $\approx 1.7$ with nuclear reaction and $\rho_{0}=10^{11} \mathrm{~g} / \mathrm{cm}^{3}$. For the sensitivity of Einstein Telescope of the type B, 
on the other hand, each value is enhanced as $164,47,46$, and 24, respectively. Obviously, for a higher value of $M_{i}$, the SNR is higher. However, the most realistic model is those with the nuclear reaction and $\rho_{0}=10^{11} \mathrm{~g} / \mathrm{cm}^{3}$ (for which $\mathrm{SNR}=1.7$ ). This implies that for the second generation detectors (advanced LIGO, advanced VIRGO, and KAGRA), it would be difficult to detect gravitational waves emitted by the collapse of a VMS of initial mass $\sim 300 M_{\odot}$ unless the collapse occurs for $D \lesssim 10 \mathrm{Mpc}$. However, with the third-generation detectors such as Einstein Telescope, these gravitational waves could be detected with SNR $\gtrsim 10$ for events with $D \lesssim 100 \mathrm{Mpc}$.

The right panel of Fig. 3 shows the spectrum for models M01, M03, M05, M07, and M08. This shows that for $\chi \gtrsim$ 0.5 , the peak amplitude depends weakly on the value of $\chi$, but for the small values of $\chi$, it decreases steeply, and in addition, the amplitude becomes steeply small with the decrease of $f$. Thus, to get a high SNR, a moderately large value of $\chi \gtrsim 0.5$ is necessary.

\section{SUMMARY AND DISCUSSION}

By new numerical-relativity simulations, we derived gravitational waveforms from a rotating VMS core collapsing to a $\mathrm{BH}$ and found that they are characterized by a ring-down oscillation of the formed $\mathrm{BH}$ in its early formation phase. For a plausible setting of the nuclear reaction and neutrino cooling, the initial BH mass is $\approx 20 M_{\odot}$ for $M_{\mathrm{CO}} \approx 150 M_{\odot}$. For the moderately rapidly rotating case, gravitational waves have a broad peak in the spectrum for $f \approx 300-600 \mathrm{~Hz}$ with $h_{\text {eff, ave }} \sim 10^{-22}$ for $D=50 \mathrm{Mpc}$, for which the SNR for the designed sensitivities of advanced LIGO and Einstein Telescope of the type $\mathrm{B}$ would be $\lesssim 2$ and 20 , respectively. Thus, it would be difficult to detect such gravitational waves by the second-generation detectors, but they will be one of the targets for the third-generation detectors.

We should keep in mind that for very massive CO cores $\left(M_{\mathrm{CO}} \gg 150 M_{\odot}\right)$, the initial $\mathrm{BH}$ mass would be much higher, say $100 M_{\odot}$. For such a case, the peak effective amplitude of gravitational waves would be $\sim 4 \times 10^{-22}$ and the corresponding characteristic frequency would be $\sim 100 \mathrm{~Hz}$ (see Fig. 33). For these gravitational waves, the SNR can be $\sim 10$ for $D=100 \mathrm{Mpc}$ for the designed sensitivity of advanced LIGO. Thus, for such VMS collapse, gravitational waves would have an SNR high enough for the detection by the second-generation detectors.

As we illustrated in Ref. [5], electromagnetic signals could be emitted after the $\mathrm{BH}$ formation in the collapse of rotating VMSs. Detection of gravitational waves will be used for constraining the sky location for the search of such electromagnetic signals, which could give important information on the $\mathrm{BH}$ formation and subsequent evolution of the system.

Acknowledgments: Numerical computations were performed on XC50 at CfCA of NAOJ and XC40 at YITP of Kyoto University. This work was supported by Grant-in-Aid for Scientific Research (Grants Nos. 16H02183, 16K17706, 16H05341, 15H00782) of Japanese MEXT/JSPS. KT was supported by the JSPS Overseas Research Fellowships.
[1] C. L. Fryer, S. E. Woosley, and A. Heger, Astrophys. J. 550, 372 (2001).

[2] A. Heger and S. E. Woosley, Astrophys. J. 567, 532 (2002).

[3] H. Umeda and K. Nomoto, Astrophys, J. 565, 385 (2002).

[4] K. Takahashi, T. Yoshida, H. Umeda, K. Sumiyoshi, and S. Yamada, Mon. Not. R. Astron. Soc. 456, 1320 (2016).

[5] H. Uchida, M. Shibata, K. Takahashi, and T. Yoshida, Astrophys. J. in press.

[6] S. C. Yoon, S. E. Woosley, and N. Langer, Astrophys. J. 725, 940 (2010); S. C. Yoon, A. Dierks, and N. Langer, Astron. Astrophys. 542, A113 (2012).

[7] S. Hild et al., Class. Quantum Grav. 28, 094013 (2011).

[8] K. Takahashi, T. Yoshida, and H. Umeda, Astrophys. J. 857, 111 (2018).

[9] M. Shibata, H. Uchida, and Y. Sekiguchi, Astrophys. J. 818, 157 (2016).

[10] M. Shibata and T. Nakamura, Phys. Rev. D 52, 5428 (1995): T. W. Baumgarte and S. L. Shapiro, Phys. Rev. D 59, 024007 (1998): M. Campanelli, C. O. Lousto, P. Marronetti, and Y. Zlochower, Phys. Rev. Lett. 96, 111101 (2006): J. G. Baker, et al., Phys. Rev. Lett. 96, 111102 (2006).
[11] M. Alcubierre, et al., Int. J. Mod. Phys. D 10 (2001), 273.

[12] M. Shibata, Prog. Theor. Phys. 104 (2000), 325; M. Shibata, Phys. Rev. D 67 (2003), 024033.

[13] T. Yamamoto, M. Shibata, and K. Taniguchi, Phys. Rev. D 78, 064054 (2008).

[14] K. Kyutoku, M. Shibata, and K. Taniguchi, Phys. Rev. D 82, 044049 (2010).

[15] N. Itoh, H. Hayashi, A. Nishikawa, and Y. Kohyama, Astrophys. J. Suppl. 102, 411 (1996).

[16] E. Berti, V. Cardoso, and A. O. Starinets, Class. Quantum. Grav. 26, 163001 (2009).

[17] G. Lovelace, M. Boyle, M. A. Scheel, and B. Szilágyi, Class. Quantum Grav. 29, 045003 (2012); D. A. Hemberger, et al., Phys. Rev. D 88, 064014 (2013).

[18] M. Shibata, Y. Sekiguchi, H. Uchida, and H. Umeda, Phys. Rev. D 94, 021501(R) (2016).

[19] https://dcc.ligo.org/cgi-bin/DocDB/ ShowDocument?docid $=2974$

[20] http://www.et-gw.eu

[21] If we further take into account the antenna pattern of the gravitational-wave detectors with respect to the injection direction of gravitational waves, the root-mean square of the actual amplitude which the detectors measure would be by a factor of $1 / \sqrt{5}$ smaller. 